\title{
Anticancer activity of 7,8-dihydroxyflavone in melanoma cells via downregulation of $\alpha$-MSH/cAMP/MITF pathway
}

\author{
DEOK YONG SIM, JAE KYUNG SOHNG and HYE JIN JUNG
}

Department of BT-Convergent Pharmaceutical Engineering, Sun Moon University, Chungnam 336-708, Republic of Korea

Received February 10, 2016; Accepted March 17, 2016

DOI: $10.3892 / o r .2016 .4825$

\begin{abstract}
Malignant melanoma is one of the most aggressive skin cancer and highly resistant to most conventional treatment. In the present study, we aimed to investigate the anticancer effects and mechanisms of action of 7,8-dihydroxyflavone (7,8-DHF), a monophenolic flavone, in melanoma cells. At concentrations not exhibiting cytotoxicity, 7,8-DHF potently inhibited growth and clonogenic survival of alpha-melanocyte stimulating hormone $(\alpha-\mathrm{MSH})$-stimulated B16F10 melanoma cells. Furthermore, it significantly blocked migration and invasion of the metastatic melanoma cells. We also observed that 7,8-DHF exhibits anti-melanogenic activity through inhibition of tyrosinase activity in $\alpha-\mathrm{MSH}$-stimulating condition. Notably, the suppressive activities of 7,8-DHF on melanoma progression were associated with the downregulation of microphthalmia-associated transcription factor (MITF) and its main downstream transcription targets, including hypoxiainducible factor $1 \alpha$ (HIF1 $\alpha)$ and c-MET, by a decrease in cyclic adenosine monophosphate (cAMP) level. In addition, combination treatment with 7,8-DHF and resveratrol, a known therapeutic agent against melanoma, had greater anticancer activities and MITF inhibition than treatment with each single agent in $\alpha$-MSH-treated B16F10 cells. Collectively, these findings may contribute to the potential application of $7,8-\mathrm{DHF}$ in the prevention and treatment of malignant melanoma.
\end{abstract}

\section{Introduction}

Malignant melanoma, a neoplasm of melanocytic origin, is the most invasive and deadly form of skin cancer. Although it comprises less than $5 \%$ of all dermatologic cancer cases, melanoma is responsible for the great majority of skin cancerrelated deaths. During the past 10 years, the incidence and

Correspondence to: Dr Hye Jin Jung, Department of BT-Convergent Pharmaceutical Engineering, Sun Moon University, 70 Sunmoon-ro 221, Tangjeong-myeon, Asan-si, Chungnam 336-708, Republic of Korea

E-mail: poka96@sunmoon.ac.kr

Key words: malignant melanoma, 7,8-dihydroxyflavone, microphthalmia-associated transcription factor, cyclic adenosine monophosphate, $\alpha$-melanocyte stimulating hormone annual mortality of melanoma has increased more rapidly than any other type of cancer $(1,2)$. Various environmental and genetic factors including ultraviolet (UV) light and oncogenic $B R A F$ mutations, respectively, have been reported to affect the development and progression of melanoma $(3,4)$. Treatment for melanoma mainly depends on the properties of the tumor and the time of diagnosis. If recognized in earlier stages, the tumor may be removed surgically. However, in the metastatic stage, melanoma becomes highly resistant to conventional therapies and nodal metastasis is associated with a poor prognosis for patients with advanced melanoma. Despite the recent FDA approvals of six drugs including three immunotherapies and three targeted therapies for the treatment of melanoma, the poor clinical prognosis and the lack of curative therapies for patients with advanced melanoma necessitate the development of new successful therapies $(5,6)$.

Microphthalmia-associated transcription factor (MITF), a master regulator of melanocyte development and differentiation, is associated with melanoma development and progression (7). MITF transcriptionally activates a number of genes that play pro-survival roles in melanoma such as cyclin dependent kinase 2 (CDK2), hypoxia-inducible factor $1 \alpha$ (HIFl $\alpha)$ and $c$-MET (8-10). The transcription and post-translational modifications of MITF gene are strongly influenced by multiple upstream pathways, including $\mathrm{c}-\mathrm{Kit}, \mathrm{Wnt} / \beta$-catenin and $\alpha$-melanocyte stimulating hormone ( $\alpha-\mathrm{MSH})(11-13)$. Activation of melanocortin 1 receptor (MC1R) by binding of $\alpha$-MSH induces cAMP production via activation of adenylyl cyclase (AC) and phosphorylates cAMP response elementbinding protein (CREB). Phosphorylated CREB directly binds to the MITF promoter region and stimulates MITF transcription (14). Recent studies have shown that $\alpha-\mathrm{MSH} / \mathrm{cAMP}$ pathway upregulates c-MET and HIF1 $\alpha$ expression, through MITF that binds and activates their promoters, thereby contributing to melanoma progression $(9,15)$. The oncogenic potential of MITF suggests that MITF inhibition may be an attractive therapeutic approach in malignant melanoma.

7,8-Dihydroxyflavone (7,8-DHF) is a monophenolic flavone with diverse biological effects (Fig. 1). It has been found to act as a small molecule agonist of tyrosine kinase receptor $B$ (TrkB) that has neurotrophic effects in various neurological diseases (16). 7,8-DHF also possesses potent antioxidant activity independent of its actions on TrkB, and thus protects against glutamate-induced excitotoxicity, 6-hydroxydopamine-induced dopaminergic neurotoxicity and 
<smiles>O=c1cc(-c2ccccc2)oc2c(O)c(O)ccc12</smiles>

Figure 1. Chemical structure of 7,8-DHF.

oxidative stress-induced genotoxicity (17-19). In addition, the anticancer effects of 7,8-DHF in human monocytic leukemia and oral squamous cancer cells have been reported $(20,21)$. However, its effect in melanoma has not been explored. In the present study, first we assessed the chemotherapeutic potential and underlying mechanisms of action of 7,8-DHF on malignant melanoma cells. Our results demonstrated that 7,8-DHF could efficiently suppress proliferation, survival, migration, invasion, and differentiation of highly metastatic melanoma cell line B16F10 via blocking $\alpha-\mathrm{MSH} / \mathrm{cAMP} / \mathrm{MITF}$ pathway. In addition, we suggest that 7,8-DHF might be applied in combination therapy with resveratrol, a known therapeutic agent against melanoma.

\section{Materials and methods}

Materials. 7,8-Dihydroxyflavone (7,8-DHF) was purchased from Tokyo Chemical Industry (Tokyo, Japan). Alphamelanocyte stimulating hormone $(\alpha-\mathrm{MSH})$, Matrigel ${ }^{\circledR}$, and Transwell $^{\circledR}$ chamber systems were obtained from SigmaAldrich (St. Louis, MO, USA), BD Biosciences (San Jose, CA, USA) and Corning Costar (Acton, MA, USA), respectively. L-3,4-dihydroxyphenylalanin (L-DOPA) was obtained from Acros Organics (Geel, Belgium). Anti-MITF, anti-HIF-1 $\alpha$, and anti-VEGF antibodies were purchased from Abcam (Cambridge, MA, USA), BD Bioscience (Bedford, MA, USA) and Santa Cruz Biotechnology (Santa Cruz, CA, USA), respectively. Anti-phospho-cMET, anti-c-MET, anti-phosphoAKT, anti-AKT, anti-phospho-ERK1/2, anti-ERK1/2, and anti- $\beta$-actin antibodies were obtained from Cell Signaling Technology (Danvers, MA, USA).

Cell culture and cell growth assay. B16F10 melanoma cells were grown in Dulbecco's modified Eagle's medium (Invitrogen, Grand Island, NY, USA) supplemented with $10 \%$ fetal bovine serum (FBS; Invitrogen). Cells were maintained at $37^{\circ} \mathrm{C}$ in a humidified $5 \% \mathrm{CO}_{2}$ incubator. For cell growth assay, B16F10 cells were plated at $2 \times 10^{3}$ cells/well in 96-well culture plates (SPL Life Sciences Co., Ltd., Gyeonggi, Korea). 7,8-DHF $(0-100 \mu \mathrm{M})$ was added to each well in the presence of $\alpha$-MSH (10 nM) and the cells were incubated for $72 \mathrm{~h}$. Cell growth was measured using a 3-(4,5-dimethylthiazol-2-yl)2,5-diphenyltetrazolium bromide (MTT) colorimetric assay.

Cell viability assay. Cell viability assay was performed using the trypan blue exclusion method. B16F10 cells were seeded at $1.5 \times 10^{4}$ cells/well in 24-well culture plates (SPL Life Sciences). 7,8-DHF (0-40 $\mu \mathrm{M})$ was added to each well in the presence of $\alpha-\mathrm{MSH}(10 \mathrm{nM})$ and the cells were incubated for up to $72 \mathrm{~h}$.
After $72 \mathrm{~h}$, the cells were stained with trypan blue and counted using a hemocytometer.

Colony formation assay. B16F10 cells were seeded at $5 \times 10^{2}$ cells/well in 6-well culture plates (SPL Life Sciences). $7,8-\mathrm{DHF}$ was treated to each well in the presence of $\alpha$-MSH $(10 \mathrm{nM})$ and the cells were incubated for 10 days until colonies were formed. Cells were fixed with $4 \%$ formaldehyde, stained with $0.25 \%$ crystal violet for $10 \mathrm{~min}$ and washed with doubledistilled water.

Wound healing assay. The confluent monolayer B16F10 cells were scratched using a tip and each well was washed with PBS to remove non-adherent cells. The cells were treated with various concentrations of 7,8-DHF in the presence of $\alpha$-MSH $(10 \mathrm{nM})$ and then incubated for up to $48 \mathrm{~h}$. The perimeter of the area with a central cell-free zone was confirmed under an optical microscope (Olympus, Center Valley, PA, USA).

Chemoinvasion assay. Cell invasion was assayed using a Transwell ${ }^{\circledR}$ chamber system with polycarbonate filter inserts with a pore size of $8.0 \mu \mathrm{m}$. The lower side of the filter was coated with $10 \mu \mathrm{l}$ gelatin $(1 \mathrm{mg} / \mathrm{ml})$ and the upper side was

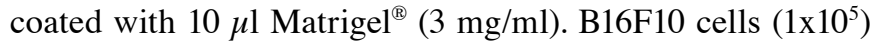
were placed in the upper chamber of the filter and 7,8-DHF was added to the lower chamber in the presence of $\alpha$-MSH $(10 \mathrm{nM})$ and $0.5 \% \mathrm{BSA}$. The chamber was incubated at $37^{\circ} \mathrm{C}$ for $24 \mathrm{~h}$, and the cells were subsequently fixed with methanol and stained with hematoxylin and eosin (H\&E). The total number of cells that invaded the lower chamber of the filter was counted using an optical microscope (Olympus).

Measurement of melanin content. B16F10 cells $\left(15 \times 10^{4}\right.$ cells/well) were plated in 12-well culture plates (SPL Life Sciences) and then treated with various concentrations of 7,8 -DHF in the presence or absence of $\alpha$-MSH $(10 \mathrm{nM})$ for $72 \mathrm{~h}$. The cells were then washed with PBS and lysed in $150 \mu \mathrm{l}$ of $1 \mathrm{M} \mathrm{NaOH}$ at $95^{\circ} \mathrm{C}$. A total of $100 \mu \mathrm{l}$ of the lysate was added in 96-well microplate and the absorbance was measured at $405 \mathrm{~nm}$ using a microplate spectrophotometer (Thermo Scientific Multiskan ${ }^{\circledR}$ Spectrum).

Determination of cellular tyrosinase activity. Tyrosinase activity was estimated by measuring the rate of L-DOPA oxidation. B16F10 cells were plated in 12-well culture plates at a density of $15 \times 10^{4}$ cells/well and then treated with various concentrations of 7,8-DHF in the presence or absence of $\alpha$-MSH (10 nM) for $72 \mathrm{~h}$. The cells were washed with PBS and lysed in $50 \mathrm{mM}$ phosphate buffer ( $\mathrm{pH}$ 6.8) containing $1 \%$ Triton X-100 and $0.1 \mathrm{mM}$ phenylmethylsulfonyl fluoride. Cellular lysates were centrifuged at $12,000 \mathrm{rpm}$ for $20 \mathrm{~min}$ at $4^{\circ} \mathrm{C}$. The supernatant was collected and the protein content was determined by the Bradford method. The cellular extract was incubated with L-DOPA $(1.25 \mathrm{mM})$ in $25 \mathrm{mM}$ phosphate buffer (pH 6.8) and the absorbance at $475 \mathrm{~nm}$ was read until the reaction has finished.

Measurement of cAMP levels. B16F10 cells were treated with various concentrations of 7,8-DHF in the presence or absence of $\alpha$-MSH (10 $\mathrm{nM}$ ) and incubated for $15 \mathrm{~min}$. The cells were lysed 

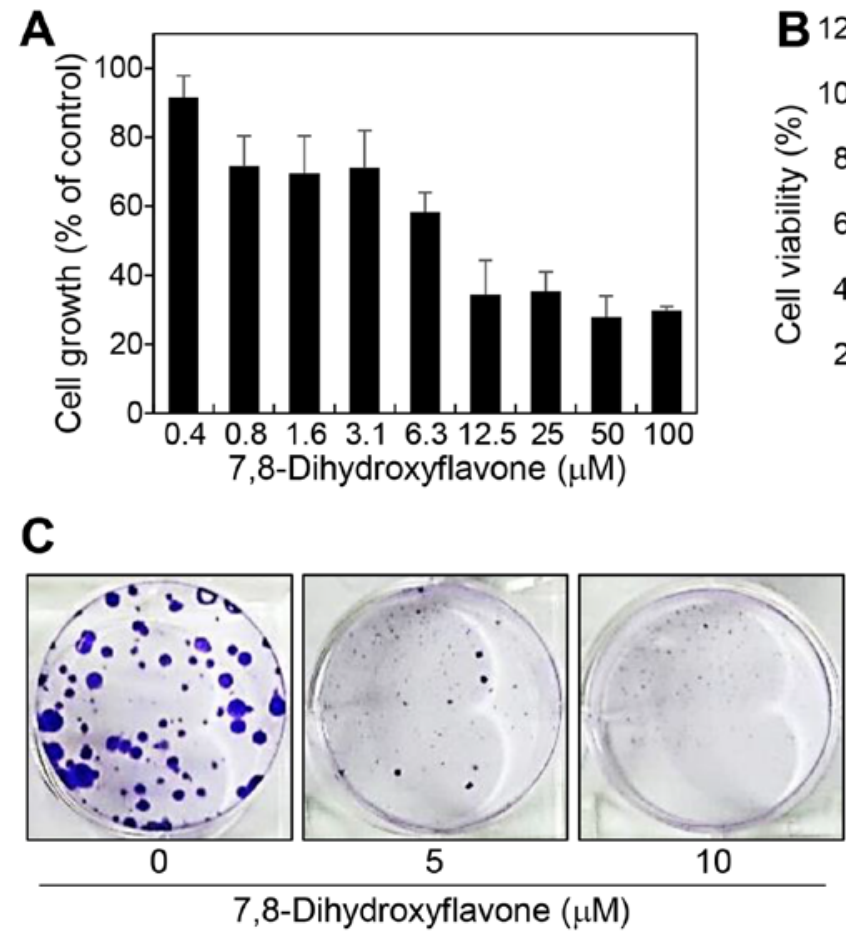
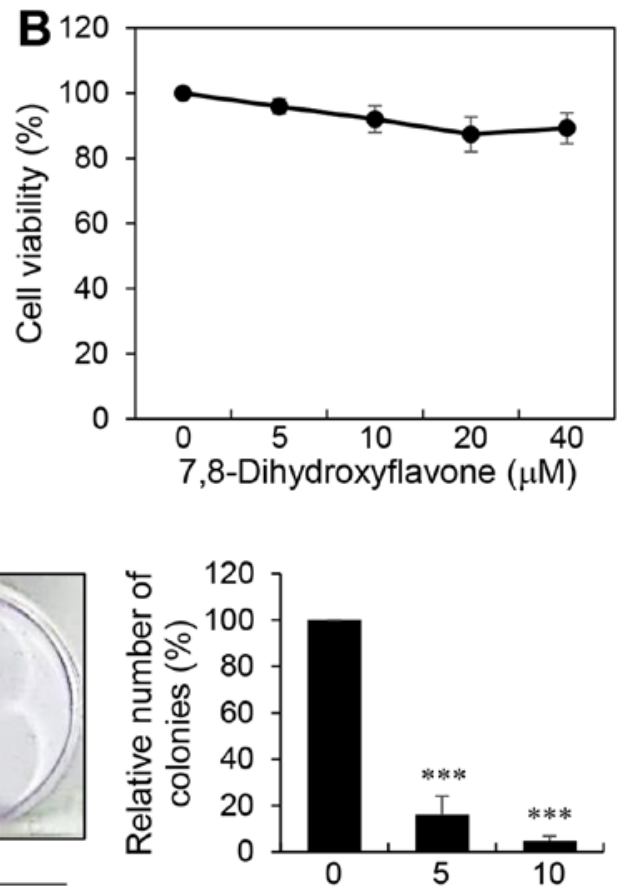

7,8-Dihydroxyflavone $(\mu \mathrm{M})$

Figure 2. The effects of 7,8-DHF on the growth and clonogenic survival of $\alpha$-MSH-stimulated B16F10 cells. (A) The effect of 7,8-DHF on the growth of B16F10. Cells were treated with various concentrations of 7,8-DHF in the presence of $\alpha-\mathrm{MSH}$ for $72 \mathrm{~h}$, and cell growth was measured using an MTT colorimetric assay. (B) The effect of 7,8-DHF on the viability of B16F10. Cells were treated with 7,8-DHF in the presence of $\alpha$-MSH and incubated for 72 h. Cell viability was measured by the trypan blue assay. (C) The effect of 7,8-DHF on the colony forming ability of B16F10. Cells were treated with 7,8-DHF in the presence of $\alpha$-MSH for 10 days. The cell colonies were visualized by crystal violet staining and then counted. ${ }^{* * * *} \mathrm{P}<0.001$ vs. control.

in $0.1 \mathrm{M} \mathrm{HCl}$, and intracellular cAMP generation was determined using enzyme-linked immunosorbent assay (ELISA) with a cAMP direct immunoassay kit (Abcam, Cambridge, MA, USA) according to the manufacturer's instructions. The cAMP levels were normalized to total protein content.

Western blot analysis. Cell lysates were separated by $10 \%$ SDS-PAGE electrophoresis and the separated proteins were transferred to polyvinylidene difluoride membranes (Millipore, Billerica, MA, USA) using standard electroblotting procedures. The blots were blocked and immunolabeled with primary antibodies against MITF, HIF-1 $\alpha$, VEGF, phosphocMET, c-MET, phospho-AKT, AKT, phospho-ERK1/2, ERK1/2 and $\beta$-actin overnight at $4^{\circ} \mathrm{C}$. Immunolabeling was detected with an enhanced chemiluminescence (ECL) kit (Bio-Rad Laboratories, Hercules, CA, USA), according to the manufacturer's instructions.

Statistical analysis. The results are expressed as the mean \pm standard error (SE). Student's t-test was used to determine statistical significance between the control and the test groups. A P-value of $<0.05$ was considered to indicate a statistically significant result.

\section{Results}

Inhibition of cell growth and clonogenic survival by 7,8-DHF in B16F10 cells. $\alpha$-MSH has been reported to elicit a variety of biological responses that bring about melanoma development as well as melanocyte differentiation (22-24).
We thus assessed the anticancer activities of 7,8-DHF against malignant melanoma in $\alpha$-MSH-stimulated B16F10 cells. To determine whether 7,8-DHF affects melanoma cell growth, B16F10 cells were treated with various concentrations of 7,8-DHF for $72 \mathrm{~h}$ and the MTT assay was performed. 7,8-DHF dose-dependently inhibited the proliferation of B16F10 with an $\mathrm{IC}_{50}$ value of $9.04 \mu \mathrm{M}$ (Fig. 2A). Notably, a trypan blue viability test revealed that 7,8 -DHF treatment did not show cytotoxic effects up to $40 \mu \mathrm{M}$ (Fig. 2B). These data indicate that the inhibition of melanoma cell growth by 7,8-DHF resulted from a cytostatic effect. To further evaluate the tumor suppressive effect of 7,8-DHF, we carried out clonogenic assay, which is an in vitro cell survival assay based on the ability of a single cell to grow into a colony. Treatment of B16F10 cells with 7,8-DHF resulted in a remarkable inhibition of the colony-forming ability (Fig. 2C). Taken together, these results suggest that 7,8-DHF may be considered a novel anticancer agent with potent antiproliferative activity against melanoma cells.

The inhibitory effects of 7,8-DHF on B16F10 cell migration and invasion. The most dangerous aspect of melanoma is its ability to spread to other parts of the body. To assess the inhibitory potential of 7,8-DHF on the metastatic ability of melanoma cells, we first confirmed its effect on migration of $\alpha$-MSH-stimulated B16F10 cells using wound healing assay. As shown in Fig. 3A, relative to untreated control cells, treatment with 7,8-DHF for $48 \mathrm{~h}$ reduced the migration capacity of B16F10 cells in a dose-dependent manner. Next, the effect of 7,8-DHF on invasive potential of $\alpha$-MSH-treated B16F10 cells 
A
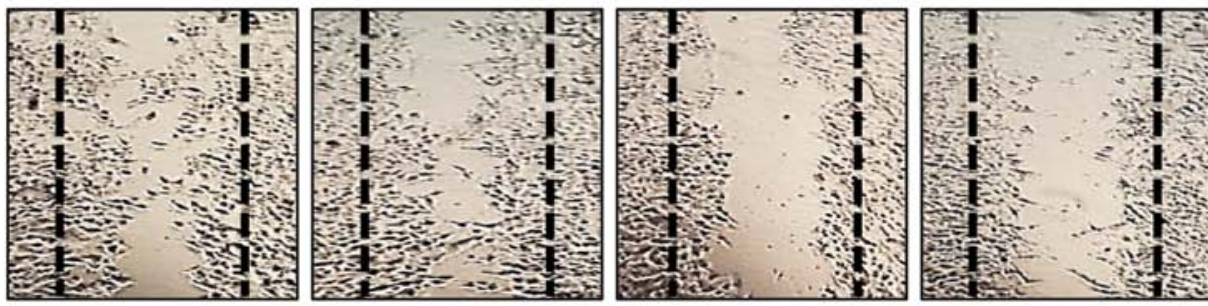

B

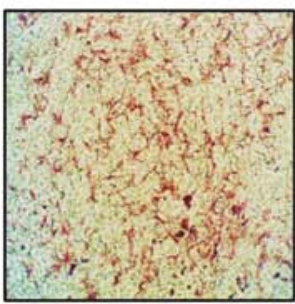

0

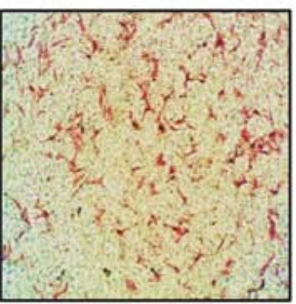

10

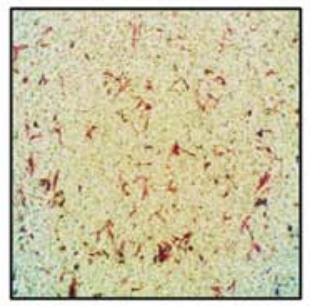

20
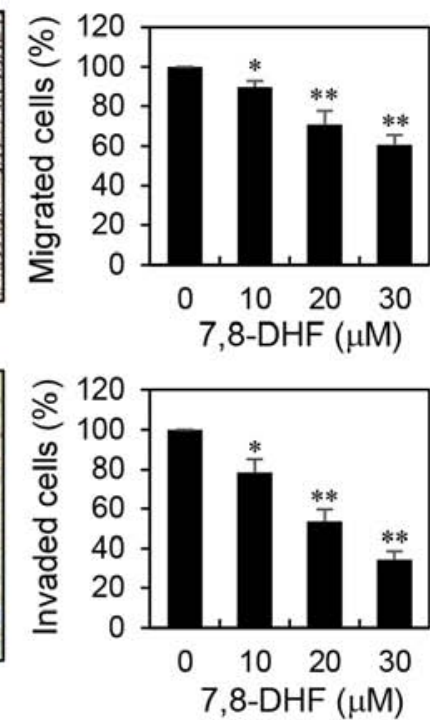

7,8-Dihydroxyflavone $(\mu \mathrm{M})$

Figure 3. The effect of 7,8-DHF on the metastatic ability of $\alpha$-MSH-stimulated B16F10 cells. (A) The effect of 7,8-DHF on the migration of B16F10. The migratory potential of B16F10 cells was analyzed using wound healing assay. Cells were stimulated with $\alpha$-MSH in the presence or absence of 7,8-DHF for $48 \mathrm{~h}$. Dotted black lines indicate the edge of the gap at $0 \mathrm{~h}$. (B) The effect of 7,8-DHF on the invasion of B16F10. The invasiveness of B16F10 cells was analyzed using Matrigel-coated polycarbonate filters. Cells were stimulated with $\alpha$-MSH in the presence or absence of 7,8-DHF for 24 h. Cells penetrating the filters were stained and counted under a light microscope. ${ }^{*} \mathrm{P}<0.05,{ }^{* *} \mathrm{P}<0.01$ vs. control.

A

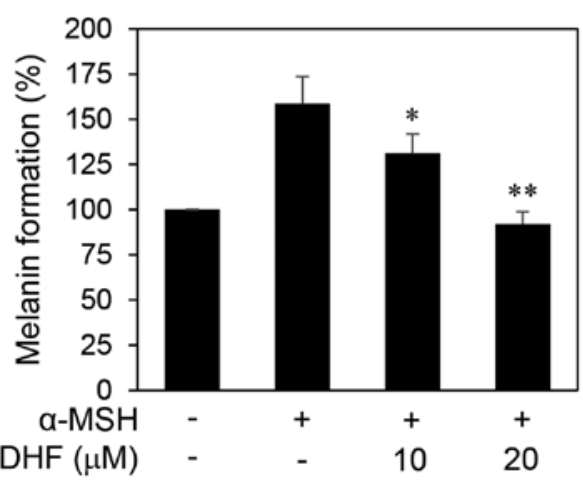

B

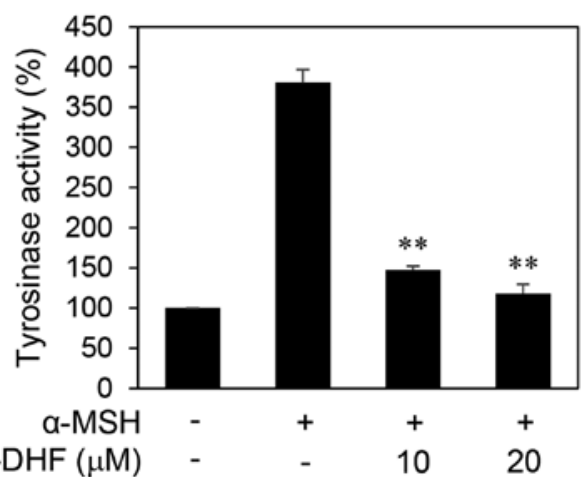

Figure 4. The effect of 7,8-DHF on the melanogenesis of $\alpha-\mathrm{MSH}$-stimulated B16F10 cells. Cells were treated with 7,8-DHF in the presence or absence of $\alpha$-MSH for $72 \mathrm{~h}$, and the cellular melanin contents (A) and tyrosinase activity (B) were determined. ${ }^{*} \mathrm{P}<0.05,{ }^{* *} \mathrm{P}<0.01 \mathrm{vs}$. the $\alpha-\mathrm{MSH}$ control.

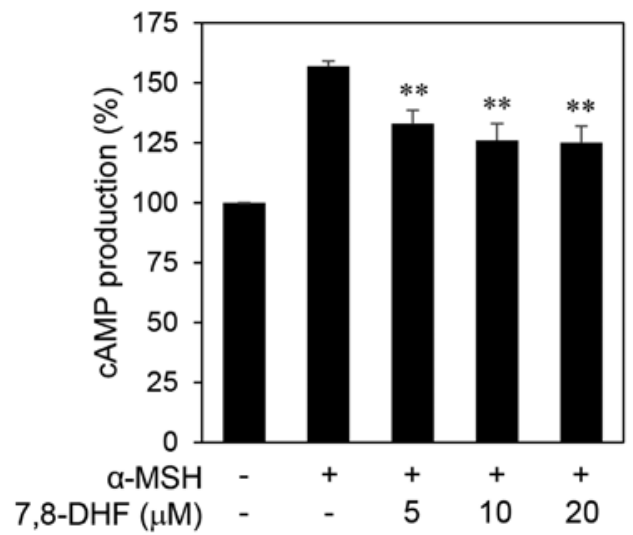

Figure 5. The effect of 7,8-DHF on $\alpha$-MSH-induced intracellular cAMP production in B16F10 cells. Cells were treated with 7,8-DHF in the presence or absence of $\alpha-\mathrm{MSH}$ and incubated for $15 \mathrm{~min}$. The cells were lysed, and intracellular cAMP levels were measured using an enzyme immunoassay protocol. ${ }^{* *} \mathrm{P}<0.01$ vs. the $\alpha-\mathrm{MSH}$ control. was investigated by employing the Matrigel matrix-coated Transwell chamber assay. As shown in Fig. 3B, 7,8-DHF treatment significantly decreased the invasiveness of B16F10 cells. These data demonstrate that 7,8-DHF has the chemotherapeutic potential to suppress melanoma metastasis.

Antimelanogenic effect of 7,8-DHF in B16F10 cells. Malignant melanocytes tend to exhibit upregulated melanogenesis and defective melanosomes (25). Cellular tyrosinase activity is the major factor that stimulates melanin synthesis and ultimately induces melanogenesis (26). We thus determined the cellular tyrosinase activity and melanin content in order to investigate the effect of 7,8-DHF on melanogenesis of B16F10 cells. The cells were stimulated by $\alpha$-MSH in the presence or absence of 7,8-DHF for 72 h. As shown in Fig. 4, treatment with 7,8-DHF clearly blocked the melanin formation and tyrosinase activity of B16F10 cells induced by $\alpha-\mathrm{MSH}$, indicating that 7,8-DHF 

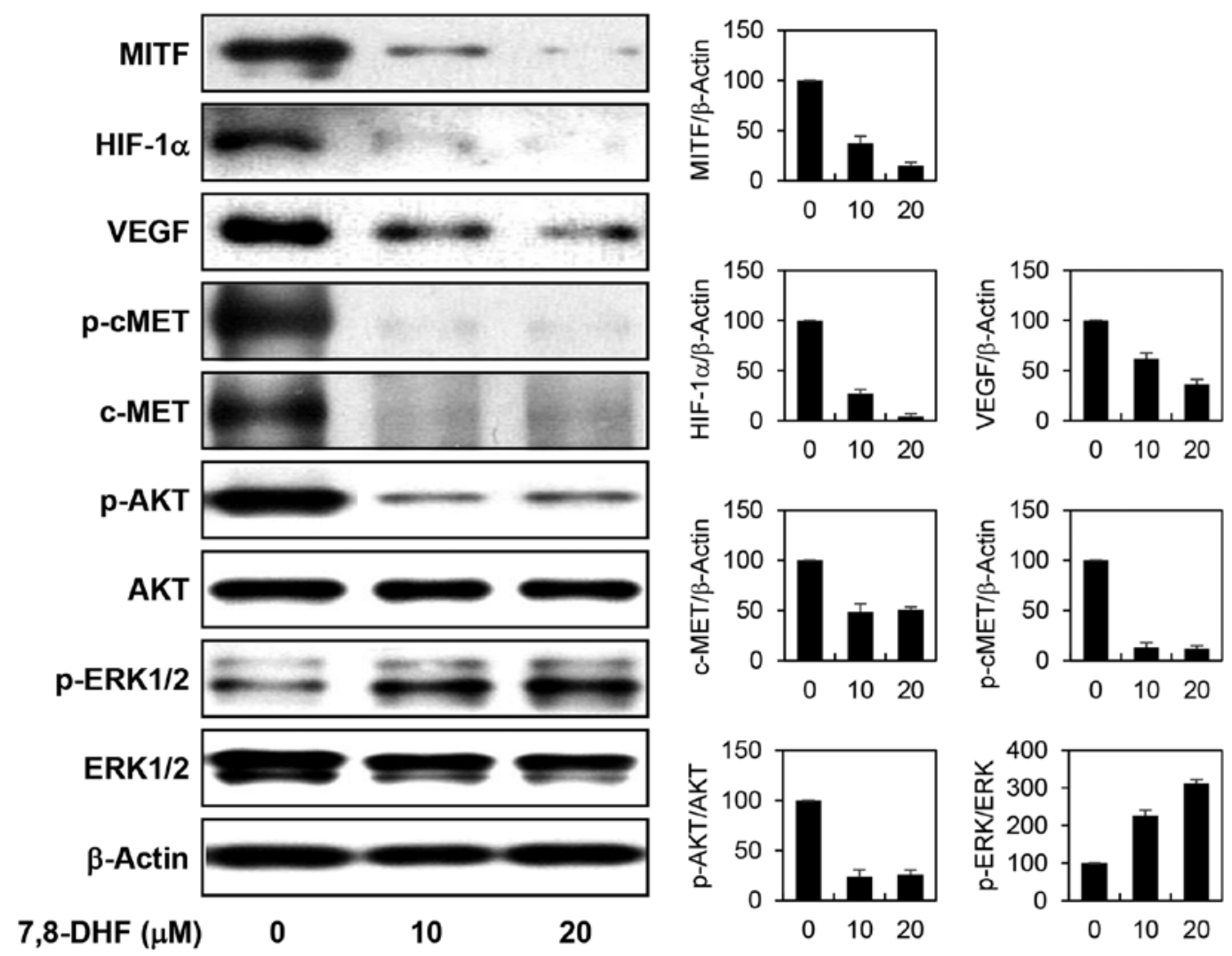

Figure 6. The effect of 7,8-DHF on the expression of MITF and its downstream transcription targets in $\alpha$-MSH-stimulated B16F10 cells. The cells were exposed to $\alpha$-MSH in the presence or absence of 7,8-DHF for $24 \mathrm{~h}$. Protein levels were detected by western blot analysis using specific antibodies and further quantified by densitometry. The level of $\beta$-actin was used as an internal control.

downregulates the differentiation of melanoma cells associated with melanogenesis.

Downregulation of MITF and its downstream transcription targets by 7,8-DHF. MITF is not only a key player in melanocyte development but is thought to also function as an oncogene in melanoma (7). $\alpha$-MSH can increase intracellular cAMP levels and consequently activate MITF expression (14). To elucidate the anticancer mechanisms of 7,8-DHF against melanoma, we investigated the effect of $7,8-\mathrm{DHF}$ on $\alpha-\mathrm{MSH} / \mathrm{cAMP} / \mathrm{MITF}$ signaling network in melanoma cells. As shown in Fig. 5, treatment with 7,8-DHF significantly reduced the intracellular cAMP levels in B16F10 cells stimulated by $\alpha$-MSH. We next examined the effect of 7,8-DHF on the expression of MITF and its downstream transcription targets. 7,8-DHF markedly decreased the protein levels of MITF in $\alpha$-MSH-stimulated B16F10 cells (Fig. 6). In addition, the expression of HIF1 $\alpha$ and c-MET, the major transcriptional targets of MITF which have been implicated in antiapoptotic and metastatic potential in melanoma, was prominently downregulated by $7,8-\mathrm{DHF}$ treatment. Furthermore, the inhibition of HIF1 $\alpha$ resulted in a remarkable reduction in expression of VEGF, its transcriptional target that is required for tumor angiogenesis. In addition, the diminished c-MET levels were also linked to the blockade of c-MET signaling such as inhibiting phosphorylation of c-MET and its downstream effector AKT. However, 7,8-DHF treatment rather increased the phosphorylation of extracellular signal-regulated kinases (ERK1/2), another c-MET downstream effector. Recent studies have revealed that activation of ERK induces phosphorylation and degradation of MITF and consequently downregulates $\alpha$-MSH-induced melanogenesis by inhibiting tyrosinase activity and expression (27,28). Accordingly, 7,8-DHF-induced activation of ERK1/2 may partly account for the antimelanogenic effect of the compound. These results collectively suggest that 7,8-DHF may exhibit the potential anticancer activity against melanoma through the downregulation of cAMP levels and subsequent suppression of the expression of MITF and its key oncogenic target genes such as HIF1 $\alpha$ and c-MET.

Enhanced anticancer effect of combination treatment with 7,8-DHF and resveratrol. Resveratrol is a naturally-occurring compound that possesses anticancer capabilities (29). A recent study demonstrated that resveratrol inhibits MITF expression, viability, and invasiveness activated by $\alpha-\mathrm{MSH}$ in melanoma cells (30). To further exploit promising combination therapies of 7,8-DHF, the anticancer effects of 7,8-DHF and resveratrol either alone or in combination were examined in $\alpha$-MSH-stimulated B16F10 cells. At the concentrations tested, combined treatment with 7,8-DHF and resveratrol more efficiently inhibited the melanoma cell growth compared with single agent treatment (growth inhibition of 43, 31 and $65 \%$ with 7,8-DHF, resveratrol, and the 7,8-DHF/resveratrol combination, respectively) (Fig. 7A). Furthermore, combination of the two compounds greatly increased suppression of the melanoma cell invasion compared to either agent alone (invasion inhibition of 28,27 and $80 \%$ with 7,8-DHF, resveratrol, and the $7,8-\mathrm{DHF} /$ resveratrol combination, respectively) 

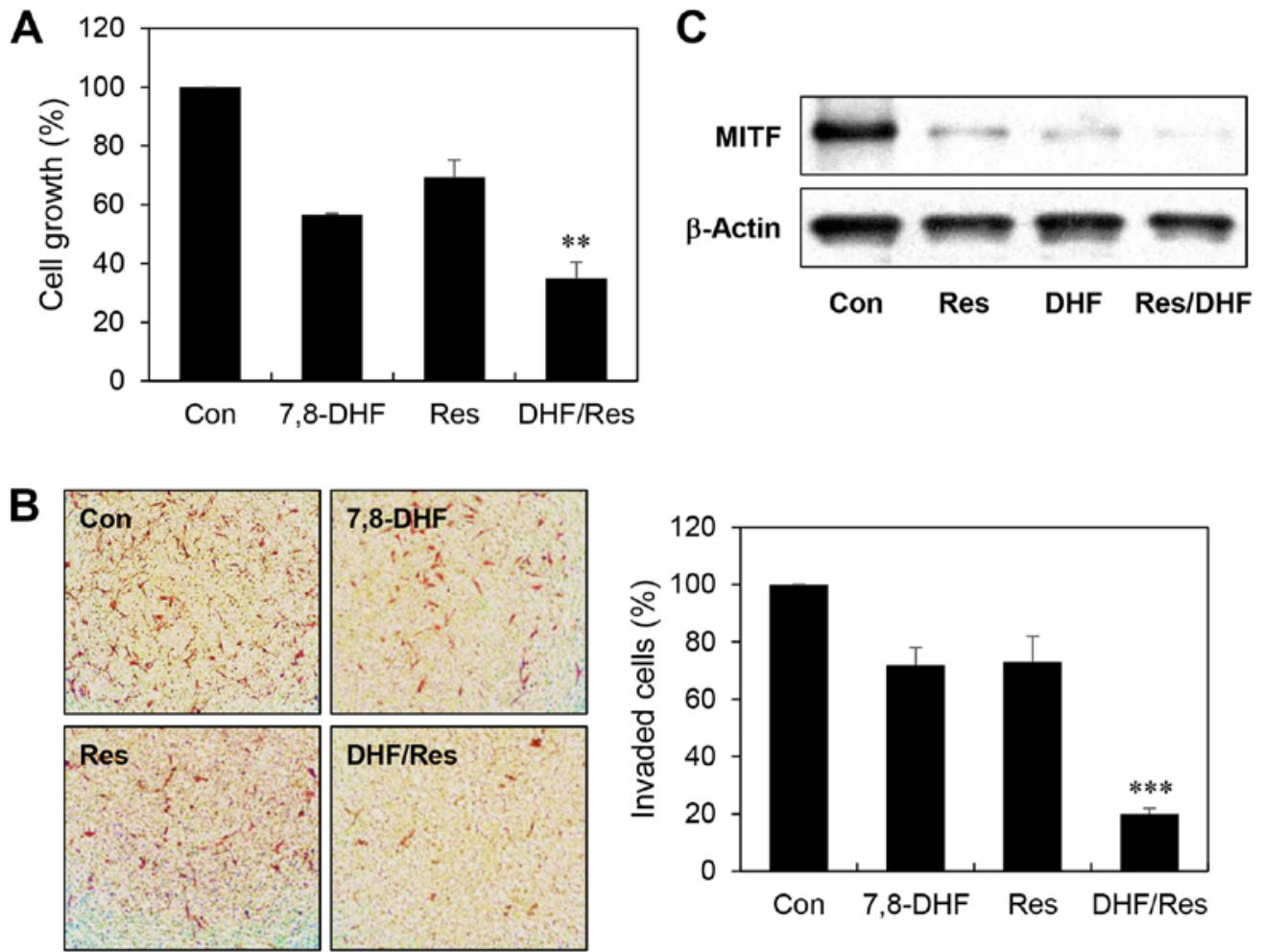

Figure 7. Enhanced anticancer effect of combination treatment with 7,8-DHF and resveratrol. B16F10 cells were exposed to $\alpha$-MSH in the presence or absence of 7,8-DHF $(10 \mu \mathrm{M})$ and resveratrol $(25 \mu \mathrm{M})$. (A) The effect of a combination of 7,8-DHF and resveratrol on the growth of B16F10. (B) The effect of combined treatment of 7,8-DHF and resveratrol on the invasiveness of B16F10. (C) The effect of combined treatment with 7,8-DHF and resveratrol on MITF expression in $\mathrm{B} 16 \mathrm{~F} 10 .{ }^{* * *} \mathrm{P}<0.01,{ }^{* * *} \mathrm{P}<0.001$ vs. single agent treatment.

(Fig. 7B). Notably, the combined treatment resulted in an enhanced reduction in MITF expression levels compared with inhibition by single agent treatment (Fig. 7C). These findings suggest that 7,8-DHF might be used to treat melanoma growth and metastasis in combination with resveratrol.

\section{Discussion}

Malignant melanoma remains one of the cancers most resistant to treatment, and the incidence and mortality rates are increasing rapidly worldwide $(1,2)$. Through our continuing efforts to discover more effective and less toxic anticancer agents for treatment of melanoma, 7,8-dihydroxyflavone (7,8-DHF), a monophenolic flavone, was newly identified as a potential anticancer compound against melanoma. Although the inhibitory effect of 7,8-DHF in some types of cancer, like human monocytic leukemia and oral squamous carcinoma $(20,21)$, has been described previously, there is no such report on melanoma so far. The key finding of the present study is that 7,8-DHF could inhibit the growth, metastasis, and melanogenesis of melanoma cells through downregulation of microphthalmia-associated transcription factor (MITF) and its related oncogenic pathways.

MITF regulates multiple targets, which are involved in various cellular processes such as cell cycle progression, survival, motility, invasion and differentiation, in melanocytes and melanoma cells (7). In human metastatic melanoma, amplification of MITF was observed and correlated with decreased overall patient survival (31). Ectopic MITF expression in conjunction with the BRAF(V600E) mutant transformed primary human melanocytes. In addition, disruption of MITF sensitized melanoma cells to chemotherapeutic agents such as docetaxel and cisplatin. Recent evidence has also shown that hypoxia-inducible factor $1 \alpha$ (HIF $1 \alpha)$ is a transcriptional target of MITF and the upregulation of $\alpha-\mathrm{MSH} / \mathrm{cAMP} / \mathrm{MITF} / \mathrm{HIF} 1$ pathway contributes to survival, neovascularization and metabolic adaptation of melanoma cells (9). Moreover, overexpression of c-MET, another target of MITF, has been associated with a poor clinical outcome in human melanoma samples. c-MET expression was increased by $\alpha-\mathrm{MSH} / \mathrm{cAMP} / \mathrm{MITF}$ pathway, resulting in invasive growth and antiapoptotic effect of melanoma cells (10). These findings demonstrate the oncogenic potential of MITF, indicating that inhibition of MITF could be an attractive therapeutic strategy to target malignant melanoma. In the present study, we found that 7,8-DHF effectively blocks $\alpha$-MSH/cAMP/MITF pathway in melanoma cells. 7,8-DHF inhibited cAMP production in $\alpha$-MSH-stimulated B16F10 cells and consequently suppressed not only MITF but also HIF1 $\alpha$ and c-MET expression, thereby inhibiting both expression of VEGF and phosphorylation of c-MET and AKT, their related oncogenic networks. In addition, the activation of ERK by 7,8-DHF is thought to cause the antimelanogenic effect in $\mathrm{B} 16 \mathrm{~F} 10$ cells by mediating downregulation of $\alpha-\mathrm{MSH} / \mathrm{cAMP} / \mathrm{MITF} / \mathrm{tyrosinase}$ pathway. These observations suggest that 7,8-DHF could be an effective chemotherapeutic agent for melanoma treatment through inhibition of MITF.

Previous studies have demonstrated that resveratrol, a natural polyphenolic compound, has both antiproliferative and proapoptotic effects in various cancer types including 
melanoma $(29,30)$. Resveratrol has been also found to sensitize several types of malignancies to anticancer drugs such as temozolomide and paclitaxel $(32,33)$. More recently, resveratrol enhanced the suppressive effect of imatinib on $\alpha-\mathrm{MSH}$ signaling, viability and invasiveness in melanoma cells (30). In the present study, we further evaluated the effect of 7,8-DHF in combination with resveratrol on melanoma cell growth and invasion. Combination treatment was more effective in inhibiting growth and invasion of $\alpha$-MSH-stimulated B16F10 cells compared to single agents. Furthermore, combination of 7,8-DHF and resveratrol was more powerful in reducing expression of MITF when compared to individual agents. Based on our data, we suggest that the use of 7,8-DHF, as single agent or in conjunction with known chemotherapeutic agents, may be a promising strategy for the management of malignant melanoma.

\section{Acknowledgements}

The present study was supported by the Sun Moon University Research Grant of 2015.

\section{References}

1. Siegel R, DeSantis C, Virgo K, Stein K, Mariotto A, Smith T, Cooper D, Gansler T, Lerro C, Fedewa S, et al: Cancer treatment and survivorship statistics, 2012. CA Cancer J Clin 62: 220-241, 2012.

2. Cummins DL, Cummins JM, Pantle H, Silverman MA, Leonard AL and Chanmugam A: Cutaneous malignant melanoma. Mayo Clin Proc 81: 500-507, 2006.

3. Kato M, Liu W, Akhand AA, Hossain K, Takeda K, Takahashi M and Nakashima I: Ultraviolet radiation induces both full activation of ret kinase and malignant melanocytic tumor promotion in RFP-RET-transgenic mice. J Invest Dermatol 115: 1157-1158, 2000.

4. Libra M, Malaponte G, Navolanic PM, Gangemi P, Bevelacqua V, Proietti L, Bruni B, Stivala F, Mazzarino MC, Travali S, et al: Analysis of BRAF mutation in primary and metastatic melanoma. Cell Cycle 4: 1382-1384, 2005.

5. Hocker TL, Singh MK and Tsao H: Melanoma genetics and therapeutic approaches in the 21st century: Moving from the benchside to the bedside. J Invest Dermatol 128: 2575-2595, 2008.

6. Tsao H, Chin L, Garraway LA and Fisher DE: Melanoma: From mutations to medicine. Genes Dev 26: 1131-1155, 2012.

7. Levy C, Khaled M and Fisher DE: MITF: Master regulator of melanocyte development and melanoma oncogene. Trends Mol Med 12: 406-414, 2006

8. Du J, Widlund HR, Horstmann MA, Ramaswamy S, Ross K, Huber WE, Nishimura EK, Golub TR and Fisher DE: Critical role of CDK2 for melanoma growth linked to its melanocytespecific transcriptional regulation by MITF. Cancer Cell 6: 565-576, 2004

9. Buscà R, Berra E, Gaggioli C, Khaled M, Bille K, Marchetti B, Thyss R, Fitsialos G, Larribère L, Bertolotto $\mathrm{C}$, et al: Hypoxia-inducible factor 1 alpha\} is a new target of microphthalmia-associated transcription factor (MITF) in melanoma cells. J Cell Biol 170: 49-59, 2005.

10. McGill GG, Haq R, Nishimura EK and Fisher DE: c-Met expression is regulated by Mitf in the melanocyte lineage. J Biol Chem 281: 10365-10373, 2006.

11. Yasumoto K, Takeda K, Saito H, Watanabe K, Takahashi K and Shibahara S: Microphthalmia-associated transcription factor interacts with LEF-1, a mediator of Wnt signaling. EMBO J 21: 2703-2714, 2002.

12. Price ER, Horstmann MA, Wells AG, Weilbaecher KN, Takemoto CM, Landis MW and Fisher DE: alpha-Melanocyte-stimulating hormone signaling regulates expression of microphthalmia, a gene deficient in Waardenburg syndrome. J Biol Chem 273: 33042-33047, 1998.
13. Wu M, Hemesath TJ, Takemoto CM, Horstmann MA, Wells AG, Price ER, Fisher DZ and Fisher DE: c-Kit triggers dual phosphorylations, which couple activation and degradation of the essential melanocyte factor Mi. Genes Dev 14: 301-312, 2000.

14. Bertolotto C, Abbe P, Hemesath TJ, Bille K, Fisher DE, Ortonne JP and Ballotti R: Microphthalmia gene product as a signal transducer in cAMP-induced differentiation of melanocytes. J Cell Biol 142: 827-835, 1998.

15. Beuret L, Flori E, Denoyelle C, Bille K, Busca R, Picardo M, Bertolotto $C$ and Ballotti R: Up-regulation of MET expression by alpha-melanocyte-stimulating hormone and MITF allows hepatocyte growth factor to protect melanocytes and melanoma cells from apoptosis. J Biol Chem 282: 14140-14147, 2007.

16. Jang SW, Liu X, Yepes M, Shepherd KR, Miller GW, Liu Y, Wilson WD, Xiao G, Blanchi B, Sun YE, et al: A selective TrkB agonist with potent neurotrophic activities by 7,8-dihydroxyflavone. Proc Natl Acad Sci USA 107: 2687-2692, 2010.

17. Chen J, Chua KW, Chua CC, Yu H, Pei A, Chua BH, Hamdy RC, $\mathrm{Xu} \mathrm{X}$ and Liu CF: Antioxidant activity of 7,8-dihydroxyflavone provides neuroprotection against glutamate-induced toxicity. Neurosci Lett 499: 181-185, 2011.

18. Zhang R, Kang KA, Piao MJ, Ko DO, Wang ZH, Chang WY, You HJ, Lee IK, Kim BJ, Kang SS, et al: Preventive effect of 7,8-dihydroxyflavone against oxidative stress induced genotoxicity. Biol Pharm Bull 32: 166-171, 2009.

19. Han X, Zhu S, Wang B, Chen L, Li R, Yao W and Qu Z: Antioxidant action of 7,8-dihydroxyflavone protects $\mathrm{PC} 12$ cells against 6-hydroxydopamine-induced cytotoxicity. Neurochem Int 64: 18-23, 2014

20. Lee RH, Shin JC, Kim KH, Choi YH, Chae JI and Shim JH: Apoptotic effects of 7,8-dihydroxyflavone in human oral squamous cancer cells through suppression of Sp1. Oncol Rep 33: 631-638, 2015.

21. Park HY, Kim GY, Hyun JW, Kim ND, Kim CG, Kim WJ, Yoo YH and Choi YH: 7,8-dihydroxyflavone induces G1 arrest of the cell cycle in U937 human monocytic leukemia cells via induction of the Cdk inhibitor p27 and downregulation of pRB phosphorylation. Oncol Rep 28: 353-357, 2012.

22. Hunt G, Todd C, Cresswell JE and Thody AJ: Alpha-melanocyte stimulating hormone and its analogue Nle4DPhe7 alpha-MSH affect morphology, tyrosinase activity and melanogenesis in cultured human melanocytes. J Cell Sci 107: 205-211, 1994.

23. Rusciano D, Lorenzoni P and Burger MM: Regulation of c-met expression in B16 murine melanoma cells by melanocyte stimulating hormone. J Cell Sci 112: 623-630, 1999.

24. Poser I and Bosserhoff AK: Transcription factors involved in development and progression of malignant melanoma. Histol Histopathol 19: 173-188, 2004.

25. Riley PA: Melanogenesis and melanoma. Pigment Cell Res 16: 548-552, 2003.

26. Costin GE and Hearing VJ: Human skin pigmentation: Melanocytes modulate skin color in response to stress. FASEB J 21: 976-994, 2007.

27. Lee JH, Jang JY, Park C, Kim BW, Choi YH and Choi BT: Curcumin suppresses alpha-melanocyte stimulating hormonestimulated melanogenesis in B16F10 cells. Int J Mol Med 26: 101-106, 2010.

28. Jin SH, Lee YY and Kang HY: Methyl-beta-cyclodextrin, a specific cholesterol-binding agent, inhibits melanogenesis in human melanocytes through activation of ERK. Arch Dermatol Res 300: 451-454, 2008.

29. Gusman J, Malonne $\mathrm{H}$ and Atassi G: A reappraisal of the potential chemopreventive and chemotherapeutic properties of resveratrol. Carcinogenesis 22: 1111-1117, 2001.

30. Chen YJ, Chen YY, Lin YF, Hu HY and Liao HF: Resveratrol inhibits alpha-melanocyte-stimulating hormone signaling, viability, and invasiveness in melanoma cells. Evid Based Complement Alternat Med 2013: 632121, 2013.

31. Garraway LA, Widlund HR, Rubin MA, Getz G, Berger AJ, Ramaswamy S, Beroukhim R, Milner DA, Granter SR, Du J, et al: Integrative genomic analyses identify MITF as a lineage survival oncogene amplified in malignant melanoma. Nature 436: 117-122, 2005 .

32. Osmond GW, Augustine CK, Zipfel PA, Padussis J and Tyler DS: Enhancing melanoma treatment with resveratrol. J Surg Res 172: 109-115, 2012.

33. Fulda S and Debatin KM: Sensitization for anticancer druginduced apoptosis by the chemopreventive agent resveratrol. Oncogene 23: 6702-6711, 2004. 